ALEA, Lat. Am. J. Probab. Math. Stat. 18, 1221-1239 (2021)

DOI: $10.30757 /$ ALEA.v18-45



\title{
On the local limit theorems for psi-mixing Markov chains
}

\section{Florence Merlevède, Magda Peligrad and Costel Peligrad}

Université Gustave Eiffel

LAMA and CNRS UMR 8050

5, Boulevard Descartes, Champs sur Marne,

Marne La Vallée, France.

E-mail address: florence.merlevede@univ-eiffel.fr

University of Cincinnati

Department of Mathematical Sciences

POBox 210025, Cincinnati, Oh 45221-0025, USA.

E-mail address: peligrm@ucmail.uc.edu , peligrc@ucmail.uc.edu

\begin{abstract}
In this paper we investigate the local limit theorem for additive functionals of a nonstationary Markov chain with finite or infinite second moment. The moment conditions are imposed on the individual summands and the weak dependence structure is expressed in terms of some uniformly mixing coefficients.
\end{abstract}

\section{Introduction}

A local limit theorem for partial sums $\left(S_{n}\right)_{n \geq 1}$ of a sequence of centered random variables is a result about the rate of convergence of the probabilities of the type $\mathbb{P}\left(a \leq S_{n} \leq b\right)$. Local limit theorems have been initially studied for the case of lattice random variables. The lattice case means that there exists $v>0$ and $a \in \mathbb{R}$ such that the values of all the variables in the sum $S_{n}$ are concentrated on the lattice $\{a+k v: k \in \mathbb{Z}\}$, whereas the nonlattice case means that no such $a$ and $v$ exists.

This type of limit theorem is a deep result, a fine scale behavior of the sums $S_{n}$ Controlling such probabilities is important for finding recurrence conditions for a random walk, as pointed out in Orey (1966) and further developed in Mineka and Silverman (1970), Mineka (1972). Theorems of this type are also useful in combinatorics. Bender (1973) considered asymptotic enumeration, Philipp (1988) considered continued fraction expansion, while Giuliano and Weber (2016) analyzed random models used in arithmetical number theory. In dynamical systems Guivarc'h and Hardy (1988), Aaronson and Denker (2001a) obtained local limit theorems for Gibbs Markov maps. For these reasons, this is an area of intense research in probability, dynamical systems, number theory and analysis.

Received by the editors June 24th, 2020; accepted April 13th, 2021.

2010 Mathematics Subject Classification. 60F05, 60J05.

Key words and phrases. Markov chains, Local limit theorem, Mixing.

Magda Peligrad is supported by the NSF grants DMS-1811373 and DMS-2054598. 
This field has a rich history, and originates in the classical De Moivre-Laplace theorem, which is 283 years old and precedes the central limit theorem. The early results deal with the behavior of $\mathbb{P}\left(S_{n}=k\right)$, where $S_{n}$ is a sum of i.i.d. Bernoulli random variables. Among early results on local limit theorems we mention works by Gnedenko (1948) and Gnedenko and Kolmogorov (1954). For historical notes we direct the reader to McDonald (2005).

This problem was intensively studied for i.i.d. sequences of random variables in further works by Shepp (1964), Stone (1965) and Feller (1967), just to name a few.

Local limit theorems for sums of independent non-identically distributed random variables serve as a basic mathematical tool in classical statistical mechanics and quantum statistics (see Khinchin, 1949; Hinčin, 1951). There are examples in the literature showing that, in the nonstationary case, the local limit theorems are more delicate than their convergence in distribution counterparts and, in general require additional assumptions. An important counterexample is given by Gamkrelidze (1964), pointing out this phenomenon for independent summands and a variety of sufficient conditions were developed over the years. We mention especially Rozanov's condition in the lattice case (see Rozanov (1957)), Statuljavičus's condition (see Statuljavičus, 1965) and the Mineka-Silverman condition in the non-lattice case (see Mineka and Silverman, 1970). They were further developed in Maller (1978) and Shore (1978). A unified discussion of these conditions can be found in Mukhin (1991).

There are also numerous more recent developments in several directions for independent structures. Dolgopyat (2016) treated the vector valued sequences of independent random variables.

Concerning dependent random variables we should mention early works on Markov chains by Kolmogorov (1962). In the lattice case, for countable state Markov chains with finite second moments, the local limit theorem is discussed in Siraždinov (1955) and Séva (1995) while the case of infinite variance is analyzed in Aaronson and Denker (2001a) and Szewczak (2008, 2010). Also in the stationary case we mention the local limit theorems for Markov chains in the papers by Hervé and Pène (2010) and Ferré et al. (2012). Hafouta and Kifer (2016) proved a local limit theorem for nonconventional sums for a class of stationary Markov chains.

Many of the results mentioned above apply to classes of uniformly mixing Markov chains, especially the class known under the name of $\psi$-mixing Markov chains. As noticed in Denker (1992) and Bryc $(1990,1992)$ the concept of $\psi$-mixing is well suited to derive large deviation results. As examples of $\psi$-mixing Markov chains we mention Gibbs-Markov dynamical systems introduced in Aaronson and Denker (2001b), which contain finite state aperiodic Markov chains and certain recurrent Markov chains with infinite state space.

In this paper we shall continue the study of the local limit theorem for a class of nonstationary $\psi$-mixing Markov chains.

We assume now that $\left(\xi_{k}\right)_{k \geq 1}$ is a Markov chain defined on $(\Omega, \mathcal{K}, \mathbb{P})$ with values in $(S, \mathcal{B}(S))$ with regular transition probabilities,

$$
Q_{k}(x, A)=\mathbb{P}\left(\xi_{k} \in A \mid \xi_{k-1}=x\right) ; \quad P_{k}(A)=\mathbb{P}\left(\xi_{k} \in A\right) \text { where } A \in \mathcal{B}(S) .
$$

Also, we denote by $Q_{k}$ the associated operator defined on $\mathbb{L}_{2}(\mathcal{B}(S))$ by $Q_{k} g(x)=\int g(y) Q_{k}(x, d y)$.

For some real-valued measurable functions $g_{j}$ on $S$ define

$$
X_{j}=g_{j}\left(\xi_{j}\right) .
$$

The sequence $\left(X_{j}\right)_{j \geq 1}$ is assumed centered $\left(\mathbb{E}\left(X_{j}\right)=0\right.$ for all $\left.j \geq 1\right)$ and, unless otherwise specified, having finite second moments $\left(\mathbb{E}\left(X_{j}^{2}\right)<\infty\right.$ for all $\left.j \geq 1\right)$. Set

$$
S_{n}=\sum_{k=1}^{n} X_{k}, \sigma_{n}^{2}=\mathbb{E}\left(S_{n}^{2}\right) \text { and } \tau_{n}^{2}=\sum_{j=1}^{n} \mathbb{E}\left(X_{j}^{2}\right) .
$$

We shall assume that there are two constant $a>0$ and $b<\infty$ with the following property: 

have

For all $k \geq 2$ there is $S_{k}^{\prime} \in \mathcal{B}(S)$ with $P_{k-1}\left(S_{k}^{\prime}\right)=1$ such that for all $A \in \mathcal{B}(S)$ and $x \in S_{k}^{\prime}$ we

Denote

$$
a P_{k}(A) \leq Q_{k}(x, A) \leq b P_{k}(A)
$$

$$
\gamma=\frac{a^{4}}{b}
$$

Clearly $b \geq 1$ and $a \leq 1$.

Throughout the paper we shall assume that $\tau_{n}^{2} \rightarrow \infty$. As we shall see latter, since we assume $a>0$, the condition $\tau_{n}^{2} \rightarrow \infty$ is equivalent to $\sigma_{n}^{2} \rightarrow \infty$ (see subsection 3.2).

In order to obtain our results we shall combine several techniques specifically designed for obtaining local limit theorems with a bound on the characteristic function using $\gamma$. More precisely, the conditions and techniques are rooted in Mineka and Silverman (1970) and Maller (1978), who treated the local limit theorem in the non-lattice setting, for sequences of independent random variables not identically distributed. We shall prove that if we assume (1.2), then the results referring to the local CLT in Maller (1978) and also in Mineka and Silverman (1970) can be extended from independent sequences of random variables to the Markovian case. Furthermore we shall also consider the situation when the variables have infinite variance and are in the domain of attraction of the normal law.

Our paper is organized as follows. In Section 2 we present the local limit theorem for nonstationary Markov chains. In Section 3 we present bounds on the characteristic function of sums, bounds of the variance of sums and proofs of the main results.

In the following sections, the notation $a(n)=o(n)$ means that $a(n) / n \rightarrow 0$ as $n \rightarrow \infty$. Also by $\Rightarrow$ we denote the convergence in distribution.

In the sequel we shall denote by $f_{k}(t)$ the Fourier transform of $X_{k}$,

$$
f_{k}(t)=f_{X_{k}}(t)=\mathbb{E}\left(\exp \left(i t X_{k}\right)\right) \text {. }
$$

\section{Results}

The first condition we shall impose is the usual Lindeberg condition needed to obtain the CLT.

Lindeberg's condition. For any $\varepsilon>0$,

$$
\frac{1}{\tau_{n}^{2}} \sum_{k=1}^{n} \mathbb{E}\left(X_{k}^{2} I\left(\left|X_{k}\right| \geq \varepsilon \tau_{n}\right)\right) \rightarrow 0 .
$$

From Gamkrelidze (1964) we know that this condition is not sufficient for a local limit theorem. We shall impose a balance-type condition involving moments of order 2 .

Condition $\mathrm{C}_{1}$. There is $0 \leq c<1$ and $\delta>0$ such that

$$
\lim \sup _{n \rightarrow \infty} \frac{\sum_{k=1}^{n} \mathbb{E}\left(X_{k}^{2} I\left(\left|X_{k}\right|>\delta\right)\right)}{\tau_{n}^{2}}<c
$$

Next condition is a nonlattice-type condition. Recall that a random variable $X$ has a nonlattice distribution is equivalent to $\left|f_{X}(t)\right|<1$ for all $t \neq 0$.

Condition $\mathrm{C}_{2}$. Assume (2.1) and for all $u \neq 0$ there is an open interval $O_{u}$ containing $u$ and a $n_{0}=n_{0}(u)$ such that for all $t \in O_{u}$ and $n>n_{0}$,

$$
\frac{1}{n} \sum_{k=1}^{n}\left|f_{k}(t)\right|^{2}<1 \text {. }
$$

Our general local limit theorem is as follows: 
Theorem 2.1. Let $\left(X_{j}\right)_{j \geq 1}$ be defined by (1.1). Assume that Conditions $C_{1}, C_{2}$, (1.2) and (2.1) are satisfied. Then, for any function $h$ on $\mathbb{R}$ which is continuous and with compact support,

$$
\lim _{n \rightarrow \infty} \sup _{u \in \mathbb{R}}\left|\sqrt{2 \pi} \sigma_{n} \mathbb{E} h\left(S_{n}-u\right)-\exp \left(-u^{2} / 2 \sigma_{n}^{2}\right) \int h(u) \lambda(d u)\right|=0
$$

where $\lambda$ is the Lebesgue measure on the real line.

It is well known that the convergence in (2.3) implies that for any $c$ and $d$ real numbers with $c<d$

$$
\lim _{n \rightarrow \infty} \sup _{u \in \mathbb{R}}\left|\sqrt{2 \pi} \sigma_{n} \mathbb{P}\left(c+u \leq S_{n} \leq d+u\right)-(d-c) \exp \left(-u^{2} / 2 \sigma_{n}^{2}\right)\right|=0 .
$$

In particular, since $\sigma_{n} \rightarrow \infty$ as $n \rightarrow \infty$, then for fixed $A>0$,

$$
\lim _{n \rightarrow \infty} \sup _{|u| \leq A}\left|\sqrt{2 \pi} \sigma_{n} \mathbb{P}\left(c+u \leq S_{n} \leq d+u\right)-(d-c)\right|=0 .
$$

If we further take $u=0$, then

$$
\lim _{n \rightarrow \infty} \sqrt{2 \pi} \sigma_{n} \mathbb{P}\left(S_{n} \in[c, d]\right)=d-c .
$$

In other words, the sequence of measures $\sqrt{2 \pi} \sigma_{n} \mathbb{P}\left(S_{n} \in[c, d]\right)$ of the interval $[c, d]$ converges to the Lebesgue measure.

As we can easily verify, condition $\mathrm{C}_{1}$ is satisfied under stronger condition: There is $0 \leq c<1$ and $\delta>0$ such that

$$
\frac{\mathbb{E}\left(X_{k}^{2} I\left(\left|X_{k}\right|>\delta\right)\right)}{\mathbb{E}\left(X_{k}^{2}\right)}<c \text { for all } k,
$$

and also under Mineka and Silverman (1970) condition, namely: For some $\delta>0$ and $d>0$,

$$
\mathbb{E}\left(X_{k}^{2} I\left(\left|X_{k}\right| \leq \delta\right)\right) \geq d \mathbb{E}\left(X_{k}^{2}\right) \text { for all } k .
$$

Clearly Condition $\mathrm{C}_{1}$ is trivially implied if there is $C>0$ such that $\left|X_{k}\right| \leq C$ a.s. for all $k$, or under the near stationarity assumption:

There is a random variable $X$ and constants $0<c_{1} \leq 1$ and $c_{2} \geq 1$ such that

$$
c_{1} \mathbb{P}(|X| \geq x) \leq \mathbb{P}\left(\left|X_{k}\right| \geq x\right) \leq c_{2} \mathbb{P}(|X| \geq x) \text { for all } x \geq 1 \text { and all } k \in \mathbb{N} .
$$

Condition $\mathrm{C}_{2}$ is satisfied in the stationary case if the marginal distribution satisfies $\left|f_{0}(t)\right|<1$ for all $t \neq 0$.

Remark 2.2. As we can notice from formula (3.1), instead of the right hand side of condition (1.2) we can use a slightly weaker version of it, namely

$$
a P_{k}(A) \leq Q_{k}(x, A) \text { and } \int Q_{k}(y, A) Q_{k-1}(x, d y) \leq b P_{k}(A)
$$

Remark 2.3. Theorem 2.1 can be reformulated for triangular arrays of Markov chains $\left(\xi_{n, i}\right)_{1 \leq i \leq n}$ and $X_{n, i}=g_{n, i}\left(\xi_{n, i}\right)$. The difference is that in condition (2.1) and in Conditions $\mathrm{C}_{1}$ and $\mathrm{C}_{2}$ the we have to replace $X_{k}$ by $X_{n, k}$ and $f_{k}$ by $f_{n, k}$. The relations in (1.2) become for $a>0$ and $b<\infty$

$$
a P_{n, k}(A)<Q_{n, k}(x, A) \leq b P_{n, k}(A) .
$$

By using Theorem 2.1 we can treat linear statistics with coefficients which are uniformly bounded above and stay away from 0 .

Corollary 2.4. Assume that $\left(\xi_{k}\right)_{k \in \mathbb{Z}}$ is a strictly stationary Markov chain. For a measurable function $g$ and $k \in \mathbb{Z}$, define $X_{k}=g\left(\xi_{k}\right)$. Assume that $\mathbb{E}\left(X_{k}\right)=0$ and $\mathbb{E}\left(X_{k}^{2}\right)<\infty$. Assume that $X_{0}$ has a non-lattice distribution and condition (1.2) is satisfied. We consider an array of real 
numbers $\left(a_{n, k}\right)_{k \geq 1}$ such that there are two positive constants $m, M$ with $0<m \leq\left|a_{n, k}\right| \leq M$ for all $n$ and $k$. Define

$$
X_{n, \ell}=a_{n, \ell} X_{\ell}
$$

Then, for any function $h$ on $\mathbb{R}$ which is continuous and with compact support, $S_{n}=\sum_{\ell=1}^{n} X_{n, \ell}$ satisfies (2.3).

Remark 2.5. Note that the strictly stationary case follows from Corollary 2.4 if we take for all $1 \leq \ell \leq n$ the constants $a_{n, \ell}=1$.

With a very similar proof as of Corollary 2.4 we can treat the linear processes with short memory.

Corollary 2.6. Let $\left(X_{k}\right)_{k \in \mathbb{Z}}$ be as in Corollary 2.4. Let $\left(a_{i}\right)_{i \geq 1}$ be a sequence of real numbers such that $\sum_{i \geq 1}\left|a_{i}\right|<\infty$ and $m=\inf _{j}\left|A_{j}\right|>0$, where $A_{j}=a_{1}+a_{2}+\ldots+a_{j}$. Construct

$$
Y_{k}=\sum_{i \geq 1} a_{i} X_{k+i} \text { and } S_{n}=\sum_{k=1}^{n} Y_{k} .
$$

Assume that $X_{0}$ has a nonlattice distribution and condition (1.2) is satisfied. Then, for any function $h$ on $\mathbb{R}$ which is continuous and with compact support,

$$
\lim _{n \rightarrow \infty} \sup _{u \in \mathbb{R}}\left|\sqrt{2 \pi} \sigma_{n}\right| A\left|\mathbb{E} h\left(S_{n}-u\right)-\exp \left(-u^{2} / 2 \sigma_{n}^{2} A^{2}\right) \int h(u) \lambda(d u)\right|=0,
$$

where $\sigma_{n}^{2}=\mathbb{E}\left(\sum_{i=1}^{n} X_{i}\right)^{2}$ and $A=\lim _{j \rightarrow \infty} A_{j}$.

We can also provide a result for the stationary situation when the variance of the individual summands can be infinite. As an application of the proof of Theorem 2.1 we obtain the following corollary:

Corollary 2.7. Assume that $\left(\xi_{k}\right)_{k \in \mathbb{Z}}$ is a strictly stationary Markov chain. Define $\left(X_{k}\right)_{k \in \mathbb{Z}}$ by $X_{k}=g\left(\xi_{k}\right)$ and assume $\mathbb{E}\left(X_{0}\right)=0$ and $H(x)=\mathbb{E}\left(X_{0}^{2} I\left(\left|X_{0}\right| \leq x\right)\right.$ is a slowly varying function as $x \rightarrow \infty$. Assume (1.2) and $X_{0}$ has a non-lattice distribution. Then there is $b_{n} \rightarrow \infty$ such that for any function $h$ on $\mathbb{R}$ which is continuous and with compact support,

$$
\lim _{n \rightarrow \infty} \sup _{u \in \mathbb{R}}\left|\sqrt{2 \pi} b_{n} \mathbb{E} h\left(S_{n}-u\right)-\exp \left(-u^{2} / 2 b_{n}^{2}\right) \int h(u) \lambda(d u)\right|=0 .
$$

As far as we know this corollary is new, though for Gibbs-Markov processes the result is provided in Aaronson and Denker (2001a) and for continued fraction processes can be found in Szewczak (2010).

On the other hand, we can start with a stationary Markov chain $\left(\xi_{k}\right)_{k \in \mathbb{Z}}$ satisfying condition (1.2) and then construct the nonstationary sequence $X_{n, k}=g_{n, k}\left(\xi_{k}\right)$ satisfying our conditions and therefore provide new results. Here below are two such examples:

Example 1. (Continued fraction expansion) For every irrational number $x$ in $(0,1)$ there is a unique sequence of positive integers $x_{1}, x_{2}, x_{3}, \ldots$ such that the following continued fraction expansion holds:

$$
x=\frac{1}{x_{1}+\frac{1}{x_{2}+\frac{1}{x_{3}+\cdots}}} .
$$

If we introduce on $[0,1]$ the Gauss probability measure with the density $f(x)=(\ln 2)^{-1}(1+x)^{-1}$, then the sequence $\left(x_{1}, x_{2}, x_{3}, \ldots\right)$ is a strictly stationary Markov chain. We know from Lemma 2.1 in Philipp (1988) that one can take for $a$ and $b$ which appear in condition (1.2) $a=0.2$ and $b=1.8$. Now we can consider $X_{k}$ defined by (1.1) as a measurable function of $x_{k}$ and apply both Corollary 2.4 and Corollary 2.7. 
Example 2. (Gibbs Markov chains). Let $S$ be a countable set, $p: S \times S \rightarrow[0,1]$ be an aperiodic, irrreducible stochastic matrix and $\pi_{s} \pi_{s}>0$ for all $s \in S, \sum_{s \in S} \pi_{s}=1$. Let $T: S^{\mathbb{N}} \rightarrow S^{\mathbb{N}}$ be the shift and define the Markov chain in a canonical way on $S^{\mathbb{N}}$ by

$$
\mathbb{P}\left(X_{1}=x_{1}, \ldots, X_{n}=x_{n}\right)=\pi_{x_{1}} p\left(x_{1}, x_{2}\right) \ldots p\left(x_{n-1}, x_{n}\right) .
$$

Let $\Omega \subset S^{\mathbb{N}}$ such that $\Omega=\left\{x \in S^{\mathbb{N}}: \mathbb{P}\left(X_{1}=x_{1}, \ldots, X_{n}=x_{n}\right)>0\right\}$. We assume that there is $M>1$ such that for all $s, t \in S$

$$
\frac{1}{M} \pi_{t} \leq p(s, t) \leq M \pi_{t}
$$

Then our condition (1.2) is satisfied and our results applied for suitable functions $g_{n, k}$. Such a chain is called Gibbs-Markov. For other examples of Gibbs Markov maps see Aaronson and Denker (2001b).

Example 3. In the context of Example 2, a fairly large class of countable state Markov processes satisfying condition (2.6) can be constructed by defining for $i, j \in \mathbb{N}^{*}$

$$
p(i, j)=\pi_{j}+\left(\delta_{i, j}-\delta_{i+1, j}\right) \varepsilon_{i},
$$

where for all $i \in \mathbb{N}^{*}, \delta_{i, i}=1$ and for $j \neq i$ we have $\delta_{i, j}=0$. We take $0 \leq \varepsilon_{i} \leq \min \left(1-\pi_{i}, \pi_{i+1}\right)$. In addition we assume that there is $M>1$ such that

$$
\varepsilon_{i} \leq(M-1) \min \left(\pi_{i}, \pi_{i+1} / M\right) .
$$

For example, let $M=2, \pi_{j}=2^{-j}$ and set $p(i, j)=2^{-j}+\left(\delta_{i, j}-\delta_{i+1, j}\right) 2^{-(3+i)}$.

\section{Proofs}

3.1. Bounds on the characteristic function. The bound on the characteristic function of a Markov chain is inspired by Lemma 1.5 in Nagaev (1961). It is given in the following proposition.

Proposition 3.1. Let $\left(X_{j}\right)_{j \geq 1}$ be defined by (1.1). Then

$$
\mid \mathbb{E}\left(\left.\exp \left(i u S_{n}\right)\right|^{4} \leq \prod_{j=1}^{n}\left[1-\frac{\gamma}{2}\left(1-\left|f_{j}(u)\right|^{2}\right)\right] .\right.
$$

For proving this proposition we need some preliminary considerations. For $u$ fixed let us introduce the operator $T_{k}=T_{u, k}$ defined on complex-valued bounded functions by:

$$
T_{k}(h)(x)=\int h(y) \exp \left(i u g_{k}(y)\right) Q_{k}(x, d y) .
$$

So

$$
T_{k}(h)\left(\xi_{k-1}\right)=\mathbb{E}\left(\left[h\left(\xi_{k}\right) \exp \left(i u X_{k}\right)\right] \mid \xi_{k-1}\right) .
$$

Notice that the values are also complex-valued bounded functions.

For an operator $T$ on $\mathbb{L}_{\infty}(S, \mathcal{B}(S))$ denote by $\|T\|=\sup _{|f|_{\infty}<1}\left|T_{k}(f)\right|_{\infty}$.

Lemma 3.2. For any $k \in N, u \in \mathbb{R}$ we have for all $k \geq 2$,

$$
\left\|T_{k-1} \circ T_{k}\right\|^{2} \leq 1-\frac{\gamma}{2}\left(1-\mid \mathbb{E}\left(\left.\exp \left(i u X_{k-1}\right)\right|^{2}\right) .\right.
$$

Proof. Without restricting the generality we shall assume that (1.2) is satisfied. Let $x \in S^{\prime}$, where $S^{\prime} \in \mathcal{B}(S)$ such that $P_{k-1}\left(S^{\prime}\right)=1$, for which condition (1.2) holds. By the definition of $T_{k}$ 's

$$
T_{k-1} \circ T_{k}(h)(x)=\int \exp \left(\operatorname{iug}_{k-1}(y)\right) \int h(z) \exp \left(i u g_{k}(z)\right) Q_{k}(y, d z) Q_{k-1}(x, d y) .
$$


Changing the order of integration

$$
\begin{aligned}
T_{k-1} \circ T_{k}(h)(x) & =\int h(z) \exp \left(\operatorname{iug}_{k}(z)\right) \int \exp \left(i u g_{k-1}(y)\right) Q_{k-1}(x, d y) Q_{k}(y, d z) \\
& =\int h(z) \exp \left(i u g_{k}(z)\right) m_{x}(d z),
\end{aligned}
$$

where, for $x$ fixed, $m_{x}$ is the measure defined on $\mathcal{B}(S)$ by

$$
m_{x}(A)=\int \exp \left(i u g_{k-1}(y)\right) Q_{k}(y, A) Q_{k-1}(x, d y) .
$$

Denote by $\operatorname{Var}\left(m_{x}\right)$ the total variation of $m_{x}$. With this notations and because $h$ is bounded by 1 ,

$$
\left|T_{k-1} \circ T_{k}(h)(x)\right| \leq \operatorname{Var}\left(m_{x}\right) .
$$

Now, in order to compute the total variation for $m_{x}$ we start from the following estimate:

$$
\begin{aligned}
& \left(\int Q_{k}(y, A) Q_{k-1}(x, d y)\right)^{2}-\left|\int \exp \left(i u g_{k-1}(y)\right) Q_{k}(y, A) Q_{k-1}(x, d y)\right|^{2} \\
= & \iint\left(1-\cos \left(u\left(g_{k-1}(y)-g_{k-1}\left(y^{\prime}\right)\right)\right) Q_{k}(y, A) Q_{k-1}(x, d y) Q_{k}\left(y^{\prime}, A\right) Q_{k-1}\left(x, d y^{\prime}\right)\right. \\
= & \iint 2 \sin ^{2}\left(\frac{u}{2}\left(g_{k-1}(y)-g_{k-1}\left(y^{\prime}\right)\right)\right) Q_{k}(y, A) Q_{k-1}(x, d y) Q_{k}\left(y^{\prime}, A\right) Q_{k-1}\left(x, d y^{\prime}\right) \\
\geq & a^{4} P_{k}^{2}(A) \iint 2 \sin ^{2}\left(\frac{u}{2}\left(g_{k-1}(y)-g_{k-1}\left(y^{\prime}\right)\right)\right) P_{k-1}(d y) P_{k-1}\left(d y^{\prime}\right) .
\end{aligned}
$$

But

So, we obtain

$$
\int Q_{k}(y, A) Q_{k-1}(x, d y)+\left|\int \exp \left(i u g_{k-1}(y)\right) Q_{k}(y, A) Q_{k-1}(x, d y)\right| \leq 2 b P_{k}(A) .
$$

$$
\begin{gathered}
\left(\int Q_{k}(y, A) Q_{k-1}(x, d y)\right)-\left|\int \exp \left(i u g_{k-1}(y)\right) Q_{k}(y, A) Q_{k-1}(x, d y)\right| \geq \\
\frac{a^{4}}{2 b} P_{k}(A) \iint 2 \sin ^{2}\left(\frac{u}{2}\left(g_{k-1}(y)-g_{k-1}\left(y^{\prime}\right)\right)\right) P_{k-1}(d y) P_{k-1}\left(d y^{\prime}\right) \\
=\frac{a^{4}}{2 b} P_{k}(A)\left(1-\left|f_{k-1}(u)\right|^{2}\right) .
\end{gathered}
$$

Therefore

$$
\left|m_{x}(A)\right| \leq \int Q_{k}(y, A) Q_{k-1}(x, d y)-\frac{\gamma}{2} P_{k}(A)\left(1-\left|f_{k-1}(u)\right|^{2}\right) .
$$

Now we consider $\left(A_{i}\right)_{i \in J}$ a finite partition of $S$, with sets in $\mathcal{B}(S)$. Then

$$
\sum_{i \in J}\left|m_{x}\left(A_{i}\right)\right| \leq 1-\frac{\gamma}{2}\left(1-\left|f_{k-1}(u)\right|^{2}\right)
$$

It follows that, for all $x \in S^{\prime}$,

$$
\operatorname{Var}\left(m_{x}\right) \leq 1-\frac{\gamma}{2}\left(1-\left|f_{k-1}(u)\right|^{2}\right),
$$

and Lemma 3.2 follows.

Proof of Proposition 3.1. Note that

$$
\mathbb{E}\left(\exp \left(i u S_{2 k}\right) \mid \xi_{0}=x\right)=T_{1} \circ T_{2} \circ \cdots \circ T_{2 k}(1)(x) .
$$

So

$$
\left|\mathbb{E}\left(\exp \left(i u S_{2 k}\right) \mid \xi_{0}\right)\right| \leq\left\|T_{1} \circ T_{2}\right\| \cdots\left\|T_{2 k-1} \circ T_{2 k}\right\| \text { a.s. }
$$


By Lemma 3.2 we have that, for $k \geq 1$,

$$
\left|\mathbb{E}\left(\exp \left(i u S_{2 k}\right) \mid \xi_{0}\right)\right|^{2} \leq \prod_{j=1}^{k}\left[1-\frac{\gamma}{2}\left(1-\left|f_{2 j-1}(u)\right|^{2}\right)\right] \text { a.s. }
$$

Also, by Lemma 3.2, for $k \geq 1$,

$$
\begin{gathered}
\left|\mathbb{E}\left(\exp \left(i u S_{2 k}\right) \mid \xi_{0}\right)\right|^{2} \leq\left\|T_{2} \circ T_{3}\right\|^{2} \cdots\left\|T_{2 k-2} \circ T_{2 k-1}\right\|^{2}\left\|T_{2 k}(1)\right\|^{2} \\
\leq \prod_{j=1}^{k}\left[1-\frac{\gamma}{2}\left(1-\left|f_{2 j}(u)\right|^{2}\right)\right] \text { a.s. }
\end{gathered}
$$

and so, by multiplying these two relations we get

$$
\left|\mathbb{E}\left(\exp \left(i u S_{2 k}\right) \mid \xi_{0}\right)\right|^{4} \leq \prod_{j=1}^{2 k}\left[1-\frac{\gamma}{2}\left(1-\left|f_{j}(u)\right|^{2}\right)\right] \text { a.s. }
$$

A similar result can be obtain for $\left|\mathbb{E}\left(\exp \left(i u S_{2 k+1}\right) \mid \xi_{0}\right)\right|^{4}$. The result in Proposition 3.1 follows.

3.2. Mixing conditions and the variance of partial sums. We shall clarify here the relation between $a$ and $b$ in condition (1.2) and several mixing coefficients for stochastic processes. Let $(\Omega, \mathcal{K}, \mathbb{P})$ be a probability space and let $\mathcal{A}, \mathcal{B}$ be two sub $\sigma$-algebras of $\mathcal{K}$. Define the maximal coefficient of correlation

$$
\rho(\mathcal{A}, \mathcal{B})=\sup _{X \in \mathbb{L}_{2}(\mathcal{A}), Y \in \mathbb{L}_{2}(\mathcal{B})}|\operatorname{corr}(X, Y)|,
$$

where $\mathbb{L}_{2}(\mathcal{A})$ is the space of random variables that are $\mathcal{A}$ measurable and square integrable.

Relevant to our paper are the lower and upper $\psi$-mixing coefficients defined by

$$
\begin{aligned}
\psi^{\prime}(\mathcal{A}, \mathcal{B}) & =\inf \frac{\mathbb{P}(A \cap B)}{\mathbb{P}(A) \mathbb{P}(B)} ; A \in \mathcal{A} \text { and } B \in \mathcal{B}, \mathbb{P}(A) \mathbb{P}(B)>0 \\
\psi^{*}(\mathcal{A}, \mathcal{B}) & =\sup \frac{\mathbb{P}(A \cap B)}{\mathbb{P}(A) \mathbb{P}(B)} ; A \in \mathcal{A} \text { and } B \in \mathcal{B}, \mathbb{P}(A) \mathbb{P}(B)>0
\end{aligned}
$$

We would also want to mention that the well-known $\psi$-mixing coefficient introduced in Blum et al. (1963) can be defined as

$$
\psi(\mathcal{A}, \mathcal{B})=\max \left[\psi^{*}(\mathcal{A}, \mathcal{B})-1,1-\psi^{\prime}(\mathcal{A}, \mathcal{B})\right]
$$

By a result of Bradley (2020) we have the following lemma, which will be useful to analyze the variance of partial sums.

Lemma 3.3. (Bradley, 2020)

$$
\rho(\mathcal{A}, \mathcal{B}) \leq 1-\psi^{\prime}(\mathcal{A}, \mathcal{B})
$$

Proof: For simplicity we denote $\rho=\rho(\mathcal{A}, \mathcal{B})$ and $\psi^{\prime}=\psi^{\prime}(\mathcal{A}, \mathcal{B})$. Without restricting the generality we assume $\psi^{\prime}>0$. By the definition of $\rho$ we have to show that, for any $X \in \mathbb{L}_{2}(\mathcal{A})$ and $Y \in \mathbb{L}_{2}(\mathcal{B})$,

$$
|\mathbb{E}(X Y)| \leq\left(1-\psi^{\prime}\right)\|X\|_{2}\|Y\|_{2} \text {. }
$$

By a measure theoretic argument, for variables with values in a separable Hilbert space, it is enough to prove this lemma for simple functions with mean zero. So, let $X=\sum_{i=1}^{n} a_{i} I\left(A_{i}\right)$ and $Y=\sum_{j=1}^{m} b_{j} I\left(B_{j}\right)$, where $A_{i} \in \mathcal{A}$ and $B_{j} \in \mathcal{B}$ are partitions of $\Omega$ and $X$ and $Y$ have mean 0 .

Denote

$$
R\left(A_{i} B_{j}\right)=\left(1-\psi^{\prime}\right)^{-1} \mathbb{P}\left(A_{i} B_{j}\right)-\left(1-\psi^{\prime}\right)^{-1} \psi^{\prime} \mathbb{P}\left(A_{i}\right) \mathbb{P}\left(B_{j}\right)
$$


and note that, by the definition of $\psi^{\prime}$, for all $i$ and $j$ we have that $R\left(A_{i} B_{j}\right) \geq 0$. Also

$$
\sum_{i=1}^{n} R\left(A_{i} B_{j}\right)=\mathbb{P}\left(B_{j}\right) \text { and } \sum_{j=1}^{m} R\left(A_{i} B_{j}\right)=\mathbb{P}\left(A_{i}\right) .
$$

Moreover, we have the decomposition

$$
\mathbb{P}\left(A_{i} B_{j}\right)=\psi^{\prime} \mathbb{P}\left(A_{i}\right) \mathbb{P}\left(B_{j}\right)+\left(1-\psi^{\prime}\right) R\left(A_{i} B_{j}\right) .
$$

Now, since $\mathbb{E}(X)=0$, clearly $\sum_{i=1}^{n} a_{i} \mathbb{P}\left(A_{i}\right)=0$ and therefore, by the above identity,

$$
\begin{aligned}
\mathbb{E}(X Y) & =\sum_{i, j} a_{i} b_{j} \mathbb{P}\left(A_{i} B_{j}\right)=\sum_{i, j} a_{i} b_{j}\left(\psi^{\prime} \mathbb{P}\left(A_{i}\right) \mathbb{P}\left(B_{j}\right)+\left(1-\psi^{\prime}\right) R\left(A_{i} B_{j}\right)\right) \\
& =\left(1-\psi^{\prime}\right) \sum_{i, j} a_{i} b_{j} R\left(A_{i} B_{j}\right) .
\end{aligned}
$$

It follows that

$$
|\mathbb{E}(X Y)| \leq\left(1-\psi^{\prime}\right) \sum_{i, j}\left|a_{i} b_{j}\right| R\left(A_{i} B_{j}\right)
$$

So, by applying Holder's inequality twice,

$$
\begin{aligned}
\sum_{i, j}\left|a_{i} b_{j}\right| R\left(A_{i} B_{j}\right) & \leq \sum_{i}\left|a_{i}\right|\left(\sum_{j} R\left(A_{i} B_{j}\right)\right)^{1 / 2}\left(\sum_{j} b_{j}^{2} R\left(A_{i} B_{j}\right)\right)^{1 / 2} \\
\leq & {\left[\sum_{i} \sum_{j}\left|a_{i}\right|^{2} R\left(A_{i} B_{j}\right)\right]^{1 / 2}\left[\sum_{i} \sum_{j}\left|b_{j}\right|^{2} R\left(A_{i} B_{j}\right)\right]^{1 / 2} } \\
& =\left[\sum_{i}\left|a_{i}\right|^{2} \mathbb{P}\left(A_{i}\right)\right]^{1 / 2}\left[\sum_{j}\left|b_{j}\right|^{2} \mathbb{P}\left(B_{j}\right)\right]^{1 / 2}=\|X\|_{2}\|Y\|_{2} .
\end{aligned}
$$

For a sequence $\mathbf{X}=\left(X_{k}\right)_{k \geq 1}$ of random variables $\psi_{k}^{\prime}(\mathbf{X})=\inf _{m \geq 1} \psi^{\prime}\left(\mathcal{F}_{1}^{m}, \mathcal{F}_{k+m}^{\infty}\right), \psi_{k}^{*}(\mathbf{X})=$ $\sup _{m \geq 1} \psi^{*}\left(\mathcal{F}_{1}^{m}, \mathcal{F}_{k+m}^{\infty}\right)$ and $\rho_{k}(\mathbf{X})=\sup _{m \geq 1} \rho\left(\mathcal{F}_{1}^{m}, \mathcal{F}_{k+m}^{\infty}\right)$, where $\mathcal{F}_{k}^{m}=\sigma\left(X_{j}, k \leq j \leq m\right)$.

For a Markov chain $\xi=\left(\xi_{k}\right)_{k \geq 1}$ the definitions simplify

$$
\begin{aligned}
& \psi_{k}^{\prime}=\inf _{m \geq 1} \psi^{\prime}\left(\sigma\left(\xi_{m}\right), \sigma\left(\xi_{k+m}\right)\right), \\
& \psi_{k}^{*}=\sup _{m \geq 1} \psi^{*}\left(\sigma\left(\xi_{m}\right), \sigma\left(\xi_{k+m}\right)\right), \rho_{k}=\sup _{m \geq 1} \rho\left(\sigma\left(\xi_{m}\right), \sigma\left(\xi_{k+m}\right)\right) .
\end{aligned}
$$

By Theorem 7.4 (a, c and d) in Bradley (2007)

$$
\begin{aligned}
\rho_{k+m} & \leq \rho_{k} \rho_{m} \\
\psi_{k+m} & \leq \psi_{k} \psi_{m} \\
1-\psi_{k+m}^{\prime} & \leq\left(1-\psi_{k}^{\prime}\right)\left(1-\psi_{m}^{\prime}\right) .
\end{aligned}
$$

So, by Theorem 7.5 in Bradley (2007) we know that:

If there is $n \geq 1$ such that $\rho_{n}<1$, then $\rho_{n} \rightarrow 0$ at least exponentially fast.

If there is $n \geq 1$ such that $\psi_{n}>0$, then $\rho_{n} \rightarrow 0$ at least exponentially fast.

If there is $n \geq 1$ such that $\psi_{n}^{\prime}>0$, then $1-\psi_{n}^{\prime} \rightarrow 0$ at least exponentially fast. 
Notice that, in terms of conditional probabilities, we also have the following equivalent definitions:

$$
\begin{aligned}
& \psi_{1}^{*}(\xi)=\sup _{k} \operatorname{ess} \sup _{x} \sup _{A \in \mathcal{B}(S)} Q_{k}(x, A) / P_{k}(A) . \\
& \psi_{1}^{\prime}(\xi)=\sup _{k} \operatorname{ess} \inf _{x} \inf _{A \in \mathcal{B}(S)} Q_{k}(x, A) / P_{k}(A) .
\end{aligned}
$$

Note that, by (1.2) we can take $a=\psi_{1}^{\prime}(\xi)>0$ and $b=\psi_{1}^{*}(\xi)<\infty$. In particular we have $1-\psi_{k}^{\prime}(\xi) \leq(1-a)^{k} \rightarrow 0$ exponentially fast. On the other hand condition (2.5) becomes in terms of $\psi$-mixing coefficients

$$
0<\psi_{1}^{\prime}(\xi) \text { and } \psi_{2}^{*}(\xi)<\infty .
$$

For a Markov chain of random variables, saying that $\psi_{1}<1$ is equivalent to $\psi_{1}^{\prime}>0$ and $\psi_{1}^{*}<2$, which implies our condition (1.2).

Therefore in all our results we can use the language of upper and lower $\psi$-mixing coefficients and use instead of (1.2) or (2.5), the mixing condition (3.3). Of course, our results also hold if $\psi_{1}<1$.

If we consider now measurable functions of a Markov chain $\mathbf{X}=\left(g\left(\xi_{k}\right)\right)_{k \geq 1}$, by the definition of the mixing coefficients, we notice that $a<\psi_{1}^{\prime}(\mathbf{X})$ and $\psi_{1}^{*}(\mathbf{X})<b$. Assume the variables are centered and have finite second moments. Recall that $\tau_{n}^{2}=\sum_{j=1}^{n} \operatorname{var}\left(X_{j}\right)$ and $\sigma_{n}^{2}=\mathbb{E}\left(S_{n}^{2}\right)$. From Proposition 13 in Peligrad (2012) we know that for functions of Markov chains

$$
\frac{1-\rho_{1}}{1+\rho_{1}} \leq \frac{\sigma_{n}^{2}}{\tau_{n}^{2}} \leq \frac{1+\rho_{1}}{1-\rho_{1}}
$$

By combining this inequality with Lemma 3.3 we obtain, for $a>0$,

$$
\frac{a}{2-a} \leq \frac{\sigma_{n}^{2}}{\tau_{n}^{2}} \leq \frac{2-a}{a}
$$

3.3. Preliminary general local CLT. Here we give a general local limit theorem. Its proof is based on the inversion formula for Fourier transform, which is a traditional argument for this type of behavior. Its statement is practically obtained by arguments in Section 4 in Hafouta and Kifer (2016).

Theorem 3.4. Assume that not all the variables have values in some fixed lattice. Assume that $b_{n} \rightarrow \infty$ and

$$
S_{n} / b_{n} \Rightarrow N(0,1)
$$

In addition, suppose that for each $L>0$

$$
\lim _{T \rightarrow \infty} \lim \sup _{n \rightarrow \infty} \int_{T \leq|u| \leq L b_{n}}\left|\mathbb{E} \exp \left(i u \frac{S_{n}}{b_{n}}\right)\right| d u=0 .
$$

Then, for any function $h$ on $\mathbb{R}$ which is continuous and with compact support,

$$
\lim _{n \rightarrow \infty} \sup _{u \in \mathbb{R}}\left|\sqrt{2 \pi} b_{n} \mathbb{E} h\left(S_{n}-u\right)-\exp \left(-u^{2} / 2 b_{n}^{2}\right) \int h(u) \lambda(d u)\right|=0 .
$$

By decomposing the integral in (3.6) into two parts, on $\left\{T \leq|u| \leq \delta b_{n}\right\}$ and on $\left\{\delta b_{n} \leq|u| \leq L b_{n}\right\}$, and changing the variable in the second integral we easily argue that in order to prove this theorem it is enough to show that for each $L$ fixed there is $0<\delta<L$ such that

$$
\left(D_{1}\right) \quad \lim _{T \rightarrow \infty} \lim _{n \rightarrow \infty} \sup \int_{T \leq|u| \leq b_{n} \delta}\left|\mathbb{E} \exp \left(i u \frac{S_{n}}{b_{n}}\right)\right| d u=0
$$

and

$$
\left(D_{2}\right) \quad \lim _{n \rightarrow \infty} b_{n} \int_{\delta<|u| \leq L}\left|\mathbb{E} \exp \left(i u S_{n}\right)\right| d u=0
$$


3.4. Proof of Theorem 2.1. We start the proof by mentioning that, by using Condition (1.1) in Maller (1978) and the Lindeberg's condition (2.1), Condition $\mathrm{C}_{1}$ can be verified under the following condition (its proof is postponed to the end of the paper in Lemma 3.8):

Condition A. There is $\delta>0$ and $n_{0} \in \mathbb{N}$ such that for $1 \leq|u| \leq \delta \tau_{n}$ and $n>n_{0}$

$$
\frac{\gamma}{8} \sum_{k=1}^{n}\left(1-\left|f_{k}\left(\frac{u}{\tau_{n}}\right)\right|^{2}\right)>g(u) \text { and } \exp (-g(u)) \text { is integrable on } \mathbb{R} \text {. }
$$

Furthermore, under the Lindeberg condition, Condition $\mathrm{C}_{2}$ implies:

Condition B. For $u \neq 0$ there is $c(u)$, an open interval $O_{u}$ containing $u$ and a $n_{0}=n_{0}(u)$ such that for all $t \in O_{u}$, and $n>n_{0}$

$$
\frac{\gamma}{8\left(\ln \tau_{n}\right)} \sum_{k=1}^{n}\left(1-\left|f_{k}(t)\right|^{2}\right) \geq c(u)>1 .
$$

In order to see that Condition $\mathrm{C}_{2}$ implies Condition $\mathrm{B}$, we note that the Lindeberg's condition (2.1) entails

$$
\lim _{n \rightarrow \infty} \frac{\tau_{n+1}}{\tau_{n}}=1
$$

This gives that, for any $d>1$ and all $n$ sufficiently large

Therefore,

$$
\frac{\tau_{n}}{\tau_{n_{0}}}=\frac{\tau_{n}}{\tau_{n-1}} \cdot \frac{\tau_{n-1}}{\tau_{n-2}} \cdots \frac{\tau_{n_{0}+1}}{\tau_{n_{0}}} \leq d^{n-n_{0}} .
$$

and then

$$
\ln \tau_{n} \leq\left(n-n_{0}\right) \ln d+\ln \tau_{n_{0}}=n \ln d+o(1)
$$

$$
\frac{1}{\ln \tau_{n}} \sum_{k=1}^{n}\left(1-\left|f_{k}(t)\right|^{2}\right) \geq \frac{1}{\ln d+o(1)}\left(1-\frac{1}{n} \sum_{k=1}^{n}\left|f_{k}(t)\right|^{2}\right) .
$$

So, (3.8) is satisfied if we show that for $|t-u| \leq \varepsilon$, we have:

$$
\frac{1}{\ln d+o(1)}\left(1-\frac{1}{n} \sum_{k=1}^{n}\left|f_{k}(t)\right|^{2}\right) \geq \frac{8}{\gamma} .
$$

This is equivalent to showing that for $t$ such that $|t-u| \leq \varepsilon$ and $n>n_{0}$ we have

$$
\frac{1}{n} \sum_{k=1}^{n}\left|f_{k}(t)\right|^{2} \leq 1-\frac{8}{\gamma} \ln d+o(1)
$$

If we select now $d>1$ close enough to 1 , we see that Condition $\mathrm{C}_{2}$ implies Condition $\mathrm{B}$.

Therefore, Theorem 2.1 will immediately follow from the following proposition:

Proposition 3.5. Let $\left(X_{j}\right)_{j \geq 1}$ be defined by (1.1). Assume that Conditions A, B, (1.2) and (2.1) are satisfied. Then (2.3) holds.

Proof: For proving this proposition we shall verify the conditions of Theorem 3.4. The first step is to obtain the CLT. With this aim, we shall apply Theorem 2.1 in Peligrad (1996). From Bradley (1997), we know that every lower $\psi$-mixing Markov chain (condition implied by $a>0$ ) satisfies a mixing condition called interlaced $\rho$-mixing, which is precisely the mixing condition we need to apply Theorem 2.1 in Peligrad (1996). Moreover, by (3.4) and the fact that $a>0$, condition $\sigma_{n}^{2} \rightarrow \infty$ is equivalent to $\tau_{n}^{2} \rightarrow \infty$. This means that the Lindeberg condition (2.1) is equivalent to

$$
\frac{1}{\sigma_{n}^{2}} \sum_{k=1}^{n} \mathbb{E}\left(X_{k}^{2} I\left(\left|X_{k}\right| \geq \varepsilon \sigma_{n}\right)\right) \rightarrow 0, \text { as } n \rightarrow \infty .
$$


Furthermore, also from (3.4), we deduce that

$$
\frac{1}{\sigma_{n}^{2}} \sum_{k=1}^{n} \mathbb{E}\left(X_{k}^{2}\right) \leq \frac{2-a}{a}
$$

and therefore, all the conditions in Theorem 2.1 Peligrad (1996) are satisfied and we obtain for this case that

$$
\frac{S_{n}}{\sigma_{n}} \Rightarrow N(0,1)
$$

An alternative way to prove the CLT is to use (3.4) and then Theorem 6.48 from Merlevède et al. (2019) in the Markov setting.

According to the discussion after the statement of Theorem 3.4, it remains to verify conditions $\left(D_{1}\right)$ and $\left(D_{2}\right)$. We begin by changing the variable in $\left(D_{1}\right)$ and, using (3.4) and the fact that $a>0$, we obtain that $\left(D_{1}\right)$ is equivalent to

$$
\lim _{T \rightarrow \infty} \lim \sup _{n \rightarrow \infty} \int_{T \leq|u| \leq \tau_{n} \delta}\left|\mathbb{E} \exp \left(i u \frac{S_{n}}{\tau_{n}}\right)\right| d u=0 .
$$

By Proposition 3.1 combined with Condition A, for any $1 \leq|u|<\delta \tau_{n}$,

$$
\begin{aligned}
\left|\mathbb{E} \exp \left(i u \frac{S_{n}}{\tau_{n}}\right)\right| & \leq \prod_{k=1}^{n}\left[1-\frac{\gamma}{2}\left(1-\left|f_{k}\left(\frac{u}{\tau_{n}}\right)\right|^{2}\right)\right]^{\frac{1}{4}} \\
& \leq \exp -\frac{\gamma}{2} \frac{1}{4} \sum_{k=1}^{n}\left(1-\left|f_{k}\left(\frac{u}{\tau_{n}}\right)\right|^{2}\right) \\
& \leq \exp (-g(u)) .
\end{aligned}
$$

Integrating both sides of this inequality on the intervals $T \leq|u| \leq \delta \tau_{n}$ we obtain

$$
\begin{aligned}
\int_{T \leq|u| \leq \delta \tau_{n}}\left|\mathbb{E} \exp \left(i u \frac{S_{n}}{\tau_{n}}\right)\right| d u & \leq \int_{T \leq|u| \leq \delta \tau_{n}} \exp (-g(u)) d u \\
& \leq \int_{|u|>T}^{\infty} \exp (-g(u)) d u
\end{aligned}
$$

Whence, taking first $\lim \sup _{n}$ and then $T \rightarrow \infty$, condition $\left(D_{1}\right)$ is verified.

We move now to verify $\left(D_{2}\right)$. Because the interval $[\delta, L]$ is compact, $\left(D_{2}\right)$ is verified if we can show that for any $|u|$ fixed in $[\delta, L]$ we can find an open interval $O_{u}$ such that

$$
\sigma_{n} \sup _{|t| \in O_{u}}\left|\mathbb{E} \exp \left(i t S_{n}\right)\right| \rightarrow 0 \text { as } n \rightarrow \infty .
$$

By using (3.4), it is enough to show that

$$
\tau_{n} \sup _{|t| \in O_{u}}\left|\mathbb{E} \exp \left(i t S_{n}\right)\right| \rightarrow 0 \text { as } n \rightarrow \infty .
$$

By Proposition 3.1, for any $t$,

$$
\begin{aligned}
\tau_{n}\left|\mathbb{E} \exp \left(i t S_{n}\right)\right| & \leq \tau_{n} \prod_{k=1}^{n}\left[1-\frac{\gamma}{2}\left(1-\left|f_{k}(t)\right|^{2}\right)\right]^{1 / 4} \\
& \leq \tau_{n} \exp \left(-\frac{a^{4}}{8 b} \sum_{k=1}^{n}\left(1-\left|f_{k}(t)\right|^{2}\right)\right) \\
& \leq \exp \left[\ln \tau_{n}-\frac{\gamma}{8} \sum_{k=1}^{n}\left(1-\left|f_{k}(t)\right|^{2}\right)\right] .
\end{aligned}
$$


Now (3.9) is satisfied, provided that

$$
\ln \tau_{n}\left(1-\inf _{|t| \in O_{u}} \frac{1}{\ln \tau_{n}} \frac{\gamma}{8} \sum_{k=1}^{n}\left(1-\left|f_{k}(t)\right|^{2}\right)\right) \rightarrow-\infty .
$$

Since $\tau_{n} \rightarrow \infty$, we obtain in this case that $\left(D_{2}\right)$ follows from Condition B.

We would like to mention that Condition B is satisfied under condition (1.3) of Mineka and Silverman (1970) (which is an adaptation of a condition due to Rozanov, 1957): For $u \neq 0$ there is an $\varepsilon=\varepsilon(u)>0$, for which

$$
\frac{1}{\ln \tau_{n}} \sum_{j=1}^{n} \mathbb{P}\left(X_{j}-a_{j} \in A(u, \varepsilon)\right) \rightarrow \infty,
$$

where $a_{i}$ is a bounded sequence of constants satisfying $\inf _{1 \leq j \leq \infty} \mathbb{P}\left(\left|X_{j}-a_{j}\right|<\delta\right)>0$ for every $\delta>0$ and $A(u, \varepsilon)=\{x:|x|<M,|x u-\pi m| \geq \varepsilon\}$, for each integer $m$ with $|m| \leq M$, where $M>0$ is fixed, large enough such that $\inf _{1 \leq j \leq \infty} \mathbb{P}\left(\left|X_{j}\right|<M\right)>0$ (the existence for such an $M$ is a part of the assumption).

The fact that condition (3.10) implies Condition B was proven by Mineka and Silverman (1970). Under condition (3.10), Mineka and Silverman (1970), on the top of page 595, showed that for each $u$ there is a positive constant $K_{u}$ independent on $k$ such that for all $t$ such that $|t-u|<\varepsilon / 4 M$, and for all $k \in \mathbb{N}$ we have

$$
\left|f_{k}(t)\right|^{2}-1 \leq-\frac{1}{4} K_{u} \varepsilon^{2} \mathbb{P}\left(X_{k} \in A(u, \varepsilon)\right) .
$$

Also, from Corollary 1 in Mineka and Silverman (1970), (3.10) can be replaced by the stronger condition: the variables $X_{k}$ 's have uniformly bounded densities, or by Corollary 2 in the same paper, (3.10) is satisfied if there are three rationally independent numbers $d_{1}, d_{2}, d_{3}$ such that $\inf _{1 \leq k \leq \infty} P\left(\left|X_{k}-d_{j}\right|<\delta\right)>0$ for $j=1,2,3$. Therefore we can make the following remark:

Remark 3.6. Theorem 2.1 also hold with Condition $\mathrm{C}_{2}$ replaced by condition (3.10).

3.5. Proof of Corollary 2.4. First of all we notice that, for any array $\mathbf{X}_{n}=\left(X_{n, k}\right)_{k}$ defined in this corollary, the mixing coefficients satisfy $a<\psi^{\prime}\left(\mathbf{X}_{n}\right)$ and $\psi^{*}\left(\mathbf{X}_{n}\right)<b$. According to Theorem 2.1, it is enough to verify Lindeberg's condition in (2.1) as well as conditions (2.4) and $\mathrm{C}_{2}$.

Note that $\tau_{n}^{2}=\sum_{k=1}^{n} a_{n, k}^{2} \mathbb{E}\left(X_{0}^{2}\right) \rightarrow \infty$ as $n \rightarrow \infty$. Hence, by stationarity, Lindeberg's condition becomes: for any $\varepsilon>0$,

$$
\frac{1}{\tau_{n}^{2}} \sum_{k=1}^{n} a_{n, k}^{2} \mathbb{E}\left(X_{0}^{2} I\left(\left|X_{0}\right| \geq \varepsilon \tau_{n}\right)\right)=\frac{\mathbb{E}\left(X_{0}^{2} I\left(\left|X_{0}\right| \geq \varepsilon \tau_{n}\right)\right)}{\mathbb{E}\left(X_{0}^{2}\right)} \rightarrow 0 \text { as } n \rightarrow \infty,
$$

On the other hand (2.4) becomes: there is $\delta>0$ such that

$$
\frac{\mathbb{E}\left(X_{0}^{2} I\left(\left|a_{n, k} X_{0}\right|>\delta\right)\right)}{\mathbb{E}\left(X_{0}^{2}\right)} \leq \frac{\mathbb{E}\left(X_{0}^{2} I\left(\left|X_{0}\right|>\delta / M\right)\right)}{\mathbb{E}\left(X_{0}^{2}\right)}<c \text { for all } k,
$$

which is obviously satisfied for $\delta$ large enough.

It remains to verify Condition $\mathrm{C}_{2}$. Fix $u \neq 0$, we have to find an open interval $O_{u}$ containing $|u|$ and a constant $c(u)$ such that for any $|t| \in U$ we have

$$
\left|\mathbb{E} \exp \left(i t a_{n, k} X_{0}\right)\right|^{2}=\left|f_{0}\left(a_{n, k} t\right)\right|^{2} \leq c(u)<1 .
$$

As a matter of fact, if $0<c<|u|<d$, then for any $t$ satisfying $0<c<|t|<d$, by the boundness of $\left(a_{n, k}\right)$, we also have $0<m c<\left|a_{n, k} t\right|<M d$. Now, since the distribution of $\xi_{0}$ is nonlattice, for any $v$ such that $0<m c \leq|v| \leq M d$ and because $f_{0}$ is continuous on the compact set $[-M d,-m c] \cup[m c, M d]$ we can find some constant $C(c, d)$ such that 


$$
\left|f_{0}(v)\right| \leq C(c, d)<1 \text {. }
$$

3.6. Proof of Corollary 2.6. By Theorem 5 in Peligrad and Utev (2006), we know that

$$
\frac{1}{v_{n}} S_{n} \Rightarrow A N(0,1) \text { as } n \rightarrow \infty,
$$

We also have

$$
\frac{\mathbb{E}\left(S_{n}^{2}\right)}{v_{n}^{2}} \rightarrow A^{2} \text { as } n \rightarrow \infty
$$

whence, by (3.4), we can find two constants $c_{1}>0$ and $c_{2}>0$ such that $c_{1} n \leq E\left(S_{n}^{2}\right) \leq c_{2} n$.

Recall that $A_{i}=a_{1}+\cdots+a_{i}$ and write

$$
S_{n}=\sum_{i \geq 2} b_{n, i} X_{i},
$$

where we used the notation $b_{n, i}=A_{i-1}$ for $2 \leq i \leq n$ and $b_{n, i}=a_{i-n}+\cdots+a_{i-1}=A_{i-1}-A_{i-n-1}$ for $i \geq n+1$.

Let $K_{n}$ be a positive integer such that $K_{n} \geq n$ and $n^{3 / 2} \sum_{\ell \geq K_{n}}\left|a_{\ell}\right| \rightarrow 0$, as $n \rightarrow \infty$. Let $\tilde{S}_{n}=\sum_{i=2}^{n+K_{n}} b_{n, i} X_{i}$. For any $t \in \mathbb{R}$

$$
\begin{aligned}
& \left|\mathbb{E}\left(\exp \left(i t S_{n}\right)\right)-\mathbb{E}\left(\exp \left(i t \tilde{S}_{n}\right)\right)\right| \leq 2|t| \mathbb{E}\left(\left|S_{n}-\tilde{S}_{n}\right|\right) \\
& \leq 2|t| \mathbb{E}\left(\left|X_{0}\right|\right) \sum_{i \geq n+K_{n}}\left|b_{n, i}\right| \leq 2 n|t| \mathbb{E}\left(\left|X_{0}\right|\right) \sum_{\ell \geq K_{n}}\left|a_{\ell}\right| .
\end{aligned}
$$

Hence, for each $L>0$ and any sequence $\left(b_{n}\right)$,

$$
\begin{aligned}
& \lim _{T \rightarrow \infty} \lim \sup _{n \rightarrow \infty} \int_{T \leq|u| \leq L b_{n}}\left|\mathbb{E} \exp \left(i u \frac{S_{n}}{b_{n}}\right)\right| d u \\
= & \lim _{T \rightarrow \infty} \lim \sup _{n \rightarrow \infty} \int_{T \leq|u| \leq L b_{n}}\left|\mathbb{E} \exp \left(i u \frac{\tilde{S}_{n}}{b_{n}}\right)\right| d u .
\end{aligned}
$$

Applying now Proposition 3.1 to $\tilde{S}_{n}=\sum_{i=2}^{n+K_{n}} b_{n, i} X_{i}$, it follows

$$
\begin{gathered}
\mid \mathbb{E}\left(\left.\exp \left(i u \tilde{S}_{n}\right)\right|^{4} \leq \prod_{j=1}^{n+K_{n}}\left[1-\frac{\gamma}{2}\left(1-\left|f\left(b_{n, j} u\right)\right|^{2}\right)\right]\right. \\
\leq \prod_{j=1}^{n-1}\left[1-\frac{\gamma}{2}\left(1-\left|f\left(A_{j} u\right)\right|^{2}\right)\right] \leq \exp \left[-\frac{\gamma}{2} \sum_{j=1}^{n-1}\left(1-\left|f\left(A_{j} u\right)\right|^{2}\right)\right] .
\end{gathered}
$$

From now on we can proceed exactly as in the proof of Corollary 2.4. Indeed, the proof is reduced to verify condition (2.1) and to establish Conditions $\mathrm{C}_{1}$ and $\mathrm{C}_{2}$ via the observation that $m<\left|A_{k}\right|<A$ for all $k \geq 1$.

3.7. Proof of Corollary 2.7. Its proof is based on the next proposition whose proof is similar to that of Proposition 3.5 and is left to the reader.

In the next proposition $\left(X_{k}\right)_{k \in \mathbb{Z}}$ is as in Theorem 2.1, with the exception that we do not assume that $X_{k}$ has finite second moment. For this case we have:

Proposition 3.7. Assume that there is a sequence of constants $b_{n} \rightarrow \infty$ such that

$$
\frac{S_{n}}{b_{n}} \Rightarrow N(0,1)
$$


Assume that Conditions $A$ and $B$ are satisfied with $\tau_{n}$ replaced by $b_{n}$ and that condition (1.2) holds. Then, for any function $h$ on $\mathbb{R}$ which is continuous and with compact support,

$$
\lim _{n \rightarrow \infty} \sup _{u \in \mathbb{R}}\left|\sqrt{2 \pi} b_{n} \mathbb{E} h\left(S_{n}-u\right)-\exp \left(-u^{2} / 2 b_{n}^{2}\right) \int h(u) \lambda(d u)\right|=0 .
$$

We should notice that Maller (1978), on the pages 106-107, verified Condition A (with $\tau_{n}$ replaced by $b_{n}$ ) under the assumptions: for every $x>0$

$$
\sup _{1 \leq j \leq n} \mathbb{P}\left(\left|X_{j}\right|>b_{n} x\right) \rightarrow 0 \text { as } n \rightarrow \infty
$$

and

Condition $\widetilde{\mathbf{A}}_{1}$. Denote

$$
V_{n}^{2}(x)=\sum_{k=1}^{n} \mathbb{E}\left[\left(X_{k}-\mathbb{E}\left(X_{k} I\left(\left|X_{k}\right| \leq x\right)\right)^{2} I\left(\left|X_{k}\right| \leq x\right)\right] .\right.
$$

There are constants $c>0, n_{0} \in \mathbb{N}$ and $\delta>0$ such that for all $n>n_{0}$ and $x>\delta$ we have

$$
\frac{x^{2} \sum_{k=1}^{n} \mathbb{P}\left(\left|X_{k}\right|>x\right)}{V_{n}^{2}(x)} \leq c .
$$

In order to prove Corollary 2.7, we shall verify the conditions in Proposition 3.7. First of all, by Lemma 3.3, we notice that we can apply Theorem 1 in Bradley (1988). Alternatively, one can also use Theorem 2.1 in Peligrad (1990). It follows that we can find a sequence of positive constants $b_{n} \rightarrow \infty$, such that

$$
\frac{S_{n}}{b_{n}} \Rightarrow N(0,1)
$$

It is well known that saying that $H(x)$ is a slowly varying function as $x \rightarrow \infty$ is equivalent to

$$
\lim _{x \rightarrow \infty} \frac{x^{2} \mathbb{P}(|X|>x)}{H(x)}=0 .
$$

Also clearly, since the variables have mean 0 , $\lim _{x \rightarrow \infty} \mathbb{E}\left(X_{0} I\left(\left|X_{0}\right|<x\right)=0\right.$, hence Condition $\widetilde{\mathrm{A}}_{1}$ is satisfied. Obviously condition (3.13) is also satisfied and these two properties are precisely what Maller (1978), on pages 107-108, used to show that Condition A is satisfied, with $\tau_{n}$ replaced by $b_{n}$.

Now by Theorem 18.1.1 in Ibragimov and Linnik (1971), $b_{n}=n^{1 / 2} h(n)$, where $h(n)$ as slowly varying at infinity. So

$$
\lim _{n \rightarrow \infty} \frac{b_{n}}{b_{n-1}}=1 .
$$

By the same type of arguments used for showing that Condition $\mathrm{C}_{2}$ implies condition $\mathrm{B}$, starting from (3.15) we show that Condition B is satisfied with $\tau_{n}$ replaced by $b_{n}$. The proof of this corollary is now complete.

\subsection{On the relation between Conditions $C_{1}$ and $A$.}

Lemma 3.8. Let $\left(X_{j}\right)_{j \geq 1}$ satisfying (3.11) and (3.13). Then Condition $C_{1}$ implies Condition A.

Proof: It is enough to show that Condition $\mathrm{C}_{1}$ implies Condition (1.2) in Maller (1978) and then apply his proof on pages 107-108. This condition makes used of the symmetrization method. We shall use the notations: $\tilde{X}_{k}=X_{k}-X_{k}^{*}$ with $\left(X_{k}^{*}\right)$ an independent copy of $\left(X_{k}\right)$. We have to verify there are constants $c, n_{0} \in \mathbb{N}$ and $\delta>0$ such that for all $n>n_{0}$ and $x>\delta$ we have

$$
\frac{x^{2} \sum_{k=1}^{n} \mathbb{P}\left(\left|\tilde{X}_{k}\right|>x\right)}{\sum_{k=1}^{n} \mathbb{E}\left(\tilde{X}_{k}^{2} I\left(\left|\tilde{X}_{k}\right| \leq x\right)\right.} \leq c .
$$


By the Markov inequality the expression in the left hand side is dominated by

$$
\frac{\sum_{k=1}^{n} \mathbb{E}\left(\tilde{X}_{k}^{2} I\left(\left|\tilde{X}_{k}\right|>x\right)\right.}{2 \tau_{n}^{2}-\sum_{k=1}^{n} \mathbb{E}\left(\tilde{X}_{k}^{2} I\left(\left|\tilde{X}_{k}\right|>x\right)\right.} .
$$

Now, by a desymmetrization argument and monotonicity this quantity is smaller than

$$
\frac{8 \sum_{k=1}^{n} \mathbb{E}\left(X_{k}^{2} I\left(\left|X_{k}\right|>x / 2\right)\right.}{2 \tau_{n}^{2}-8 \sum_{k=1}^{n} \mathbb{E}\left(X_{k}^{2} I\left(\left|X_{k}\right|>x / 2\right)\right.},
$$

which is uniformly bounded under Condition $\mathrm{C}_{1}$.

3.9. Triangular arrays. A second look. In our Remark 2.3 we have already mentioned an extension to a triangular array $\left(\xi_{n, i}\right)_{1 \leq i \leq n}$ of Markov chains and $X_{n, i}=g_{n, i}\left(\xi_{n, i}\right)$. Now we discuss the situation when the mixing coefficients are different from line to line, i.e. for $a_{n}>0$ and $b_{n}<\infty$

$$
a_{n} P_{n, k}(A) \leq Q_{n, k}(x, A) \leq b_{n} P_{n, k}(A),
$$

where $P_{n, k}(A)=\mathbb{P}\left(\xi_{n, k} \in A\right)$ and $Q_{n, k}(x, A)=\mathbb{P}\left(\xi_{n, k+1} \in A \mid \xi_{n, k}=x\right)$. A natural problem is to ask if we can allow $a_{n} \rightarrow 0$. This question is motivated by a remarkable result concerning the central limit theorem for triangular arrays of Markov chains, which is due to Dobrušin $(1956 \mathrm{a}, \mathrm{b})$ and further developed in several papers including Gudinas (1977) and Peligrad (2012). Actually, the mixing coefficient needed for a CLT is more general, larger than $a_{n}$ in (3.16). Practically, a CLT holds under the condition:

$$
\frac{1}{a_{n} \tau_{n}^{2}} \sum_{k=1}^{n} \mathbb{E}\left(X_{n, k}^{2} I\left(\left|X_{n, k}\right| \geq \varepsilon a_{n} \tau_{n}\right)\right) \rightarrow 0 \text { as } n \rightarrow \infty .
$$

By using the proof of Proposition 3.5, we can give the following technical sufficient conditions for the local limit theorem with mixing coefficients which are not uniformly bounded.

Theorem 3.9. Assume that $\left(\xi_{n, i}\right)_{1 \leq i \leq n}$ is a triangular array of Markov chains and $X_{n, i}=g_{n, i}\left(\xi_{n, i}\right)$, where $\mathbb{E}\left(X_{n, i}\right)=0, \mathbb{E}\left(X_{n, i}^{2}\right)<\infty$ and condition (3.17) is satisfied. Assume $a_{n} \tau_{n}^{2} \rightarrow \infty$ and there is $\delta>0$ and $n_{0} \in \mathbb{N}$ such that for $1 \leq|u| \leq \delta \tau_{n}$ and $n>n_{0}$

$$
\frac{a_{n}^{4}}{8 b_{n}} \sum_{k=1}^{n}\left(1-\left|f_{n, k}\left(\frac{u}{\tau_{n}}\right)\right|^{2}\right)>g(u) \text { and } \exp (-g(u)) \text { is integrable on } \mathbb{R} \text {. }
$$

Also assume that for $u \neq 0$ there is $c(u)$, an open interval $O_{u}$ containing $u$ and $a n_{0}=n_{0}(u)$ such that for all $t \in O_{u}$, and $n>n_{0}$

$$
\frac{a_{n}^{4}}{8\left(\ln \tau_{n}\right) b_{n}} \sum_{k=1}^{n}\left(1-\left|f_{n, k}(t)\right|^{2}\right) \geq c(u)>1 .
$$

Then (2.3) holds.

In particular, this theorem can be applied to row-wise stationary triangular arrays. They are useful to study the local stationary Markov chains as defined, for instance, in Truquet (2019).

\section{Acknowledgements}

This paper was developed concomitantly and independently of a new research monograph by Dolgopyat and Sarig (2020), which treats the local limit theorem for a different nonstationary situation. There are many differences between our paper and this monograph. For instance there are differences with respect to the mixing conditions. They used a condition of type (2.5) but with reverse inequalities. Nevertheless, in both papers the mixing conditions imply $\psi$-mixing. We treat instantaneous functions of a Markov chain, while Dolgopyat and Sarig look at functions of two 
consecutive random variables. However, the main difference is that Dolgopyat and Sarig (2020) deal with bounded random variables and we used finite (or even infinite) second moment conditions.

The authors would like to thank Richard Bradley for the statement and proof of Lemma 3.3 and to Jon Aaronson for useful discussions. The authors would also like to thank the two referees for their very valuable comments, which improved the presentation of this paper.

\section{References}

Aaronson, J. and Denker, M. A local limit theorem for stationary processes in the domain of attraction of a normal distribution. In Asymptotic methods in probability and statistics with applications (St. Petersburg, 1998), Stat. Ind. Technol., pp. 215-223. Birkhäuser Boston, Boston, MA (2001a). MR1890328.

Aaronson, J. and Denker, M. Local limit theorems for partial sums of stationary sequences generated by Gibbs-Markov maps. Stoch. Dyn., 1 (2), 193-237 (2001b). MR1840194.

Bender, E. A. Central and local limit theorems applied to asymptotic enumeration. J. Combinatorial Theory Ser. A, 15, 91-111 (1973). MR375433.

Blum, J. R., Hanson, D. L., and Koopmans, L. H. On the strong law of large numbers for a class of stochastic processes. Z. Wahrscheinlichkeitstheorie und Verw. Gebiete, 2, 1-11 (1963). MR161369.

Bradley, R. C. A central limit theorem for stationary $\rho$-mixing sequences with infinite variance. Ann. Probab., 16 (1), 313-332 (1988). MR920274.

Bradley, R. C. Every "lower psi-mixing" Markov chain is "interlaced rho-mixing". Stochastic Process. Appl., 72 (2), 221-239 (1997). MR1486554.

Bradley, R. C. Introduction to strong mixing conditions. Vol. 2. Kendrick Press, Heber City, UT (2007). ISBN 0-9740427-7-3. MR2325295.

Bradley, R. C. A relation between' $\rho$ and lower $\psi$-mixing coefficients. Personal communication (2020).

Bryc, W. o. Large deviations by the asymptotic value method. In Diffusion processes and related problems in analysis, Vol. I (Evanston, IL, 1989), volume 22 of Progr. Probab., pp. 447-472. Birkhäuser Boston, Boston, MA (1990). MR1110177.

Bryc, W. o. On large deviations for uniformly strong mixing sequences. Stochastic Process. Appl., 41 (2), 191-202 (1992). MR1164173.

Denker, M. Large deviations and the pressure function. In Transactions of the 11th Prague Conference on Information Theory, Statistical Decision Functions, Random Processes, pp. 21-33. Academia Publ. House of the Czechoslovak Acad. Science. (1992).

Dobrušin, R. L. Central limit theorem for non-stationary Markov chains. I. Teor. Veroyatnost. $i$ Primenen., 1, 72-89 (1956a). MR0086436.

Dobrušin, R. L. Central limit theorem for nonstationary Markov chains. II. Teor. Veroyatnost. $i$ Primenen., 1, 365-425 (1956b). MR0097112.

Dolgopyat, D. A local limit theorem for sums of independent random vectors. Electron. J. Probab., 21, Paper No. 39, 15 (2016). MR3515569.

Dolgopyat, D. and Sarig, O. Local limit theorems for inhomogeneous Markov chains (2020). Available at https://www.math.umd.edu/dolgop/MC-LLT-Monograph5.pdf.

Feller, W. On regular variation and local limit theorems. In Proc. Fifth Berkeley Sympos. Math. Statist. and Probability (Berkeley, Calif., 1965/66), Vol. II: Contributions to Probability Theory, Part 1, pp. 373-388. Univ. California Press, Berkeley, Calif. (1967). MR0219117.

Ferré, D., Hervé, L., and Ledoux, J. Limit theorems for stationary Markov processes with $L^{2}$-spectral gap. Ann. Inst. Henri Poincaré Probab. Stat., 48 (2), 396-423 (2012). MR2954261.

Gamkrelidze, N. G. On a local limit theorem for lattice random variables. Teor. Verojatnost. $i$ Primenen, 9, 733-736 (1964). MR0177433. 
Giuliano, R. and Weber, M. Local limit theorems in some random models from number theory. Stoch. Anal. Appl., 34 (6), 941-960 (2016). MR3544164.

Gnedenko, B. V. On a local limit theorem of the theory of probability. Uspehi Matem. Nauk (N. S.), 3 (3(25)), 187-194 (1948). MR0026275.

Gnedenko, B. V. and Kolmogorov, A. N. Limit distributions for sums of independent random variables. Addison-Wesley Publishing Company, Inc., Cambridge, Mass. (1954). MR0062975.

Gudinas, P. P. An invariance principle for inhomogeneous Markov chains. Litovsk. Mat. Sb., 17 (2), 63-73, 219 (1977). MR0488304.

Guivarc'h, Y. and Hardy, J. Théorèmes limites pour une classe de chaînes de Markov et applications aux difféomorphismes d'Anosov. Ann. Inst. H. Poincaré Probab. Statist., 24 (1), 73-98 (1988). MR937957.

Hafouta, Y. and Kifer, Y. A nonconventional local limit theorem. J. Theoret. Probab., 29 (4), 1524-1553 (2016). MR3571253.

Hervé, L. and Pène, F. The Nagaev-Guivarc'h method via the Keller-Liverani theorem. Bull. Soc. Math. France, 138 (3), 415-489 (2010). MR2729019.

Hinčin, A. Y. Matematičeskie osnovaniya kvantovou statistiki. Gosudarstv. Izdat. Tehn.-Teor. Lit., Moscow-Leningrad (1951). MR0047561.

Ibragimov, I. A. and Linnik, Y. V. Independent and stationary sequences of random variables. Wolters-Noordhoff Publishing, Groningen (1971). MR0322926.

Khinchin, A. I. Mathematical Foundations of Statistical Mechanics. Dover Publications, Inc., New York, N. Y. (1949). MR0029808.

Kolmogorov, A. N. A local limit theorem for Markov chains. In Select. Transl. Math. Statist. and Probability, Vol. 2, pp. 109-129. American Mathematical Society, Providence, R.I. (1962). MR0150810.

Maller, R. A. A local limit theorem for independent random variables. Stochastic Process. Appl., 7 (1), 101-111 (1978). MR492695.

McDonald, D. R. The Local Limit Theorem: A Historical Perspective. Journal of the Iranian Statistical Society, 4 (2), 73-86 (2005). URL http://jirss.irstat.ir/article-1-127-en. html.

Merlevède, F., Peligrad, M., and Utev, S. Functional Gaussian approximation for dependent structures, volume 6 of Oxford Studies in Probability. Oxford University Press, Oxford (2019). ISBN 978-0-19-882694-1. MR3930596.

Mineka, J. Local limit theorems and recurrence conditions for sums of independent integer-valued random variables. Ann. Math. Statist., 43, 251-259 (1972). MR314096.

Mineka, J. and Silverman, S. A local limit theorem and recurrence conditions for sums of independent non-lattice random variables. Ann. Math. Statist., 41, 592-600 (1970). MR256447.

Mukhin, A. B. Local limit theorems for lattice random variables. Teor. Veroyatnost. i Primenen., 36 (4), 660-674 (1991). MR1147168.

Nagaev, S. V. More exact limit theorems for homogeneous Markov chains. Teor. Verojatnost. $i$ Primenen., 6, 67-86 (1961). MR0131291.

Orey, S. Tail events for sums of independent random variables. J. Math. Mech., 15, 937-951 (1966). MR0202178.

Peligrad, M. On Ibragimov-Iosifescu conjecture for $\phi$-mixing sequences. Stochastic Process. Appl., 35 (2), 293-308 (1990). MR1067114.

Peligrad, M. On the asymptotic normality of sequences of weak dependent random variables. $J$. Theoret. Probab., 9 (3), 703-715 (1996). MR1400595.

Peligrad, M. Central limit theorem for triangular arrays of non-homogeneous Markov chains. Probab. Theory Related Fields, 154 (3-4), 409-428 (2012). MR3000551.

Peligrad, M. and Utev, S. Invariance principle for stochastic processes with short memory. In High dimensional probability, volume 51 of IMS Lecture Notes Monogr. Ser., pp. 18-32. Inst. Math. 
Statist., Beachwood, OH (2006). MR2387758.

Philipp, W. Limit theorems for sums of partial quotients of continued fractions. Monatsh. Math., 105 (3), 195-206 (1988). MR939942.

Rozanov, Y. A. On a local limit theorem for lattice distributions. Teor. Veroyatnost. i Primenen., 2, 275-281 (1957). MR0092264.

Séva, M. On the local limit theorem for non-uniformly ergodic Markov chains. J. Appl. Probab., 32 (1), 52-62 (1995). MR1316793.

Shepp, L. A. A local limit theorem. Ann. Math. Statist., 35, 419-423 (1964). MR166817.

Shore, T. R. On the local limit theorem for independent nonlattice random variables. Ann. Probab., 6 (4), 563-573 (1978). MR496406.

Siraždinov, S. H. Limit theorems for stationary Markov chains. Izdat. Akad. Nauk Uzbekskoŭ SSR, Taškent (1955). MR0084904.

Statuljavičus, V. A. Limit theorems for densities and the asymptotic expansions for distributions of sums of independent random variables. Teor. Verojatnost. i Primene, 10, 645-659 (1965). MR0193660.

Stone, C. A local limit theorem for nonlattice multi-dimensional distribution functions. Ann. Math. Statist., 36, 546-551 (1965). MR175166.

Szewczak, Z. S. Large deviations in operator form. Positivity, 12 (4), 631-641 (2008). MR2448752.

Szewczak, Z. S. A local limit theorem for continued fractions. Stoch. Dyn., 10 (3), 429-439 (2010). MR2671385.

Truquet, L. Local stationarity and time-inhomogeneous Markov chains. Ann. Statist., 47 (4), 2023-2050 (2019). MR3953443. 